\title{
Diversidade Sexual
}

\section{Sexual Diversity}

\section{RESUMO}

As mudanças no campo da sexualidade, durante o século XX, caracterizaram-se pela influência da tecnologia, por progressos na área da Sociologia, Pedagogia e pelo advento da psicanálise. Pesquisas sobre o comportamento sexual representaram ruptura com estudos da sexologia clínica. As palavras sexo e gênero foram, e ainda são, utilizadas como sinônimos, embora pertencer a determinado sexo signifique ser homem ou mulher, biologicamente, enquanto gênero define o masculino e o feminino, do ponto de vista comportamental e psicológico. Por outro lado, o comportamento de gênero assume diferentes significados, conforme a cultura em que se insere. A expressão da sexualidade, mais do que se manifestar por diferentes heranças biológicas, também se constitui num meio de confirmação de regras sociais, o que lhe credita caráter ideológico. Técnicas de imagem cerebral ampliaram o conhecimento das diferenças do cérebro masculino e feminino. Estudos demonstraram que influências associadas ao sexo se dão em vários níveis do sistema nervoso, do genético ao comportamental, resultando em distintas organizações cerebrais, masculina e feminina, dissociadas dos estereótipos. O legado de tais mudanças é sem precedentes para a civilização ocidental. Perplexidade, estigmatização, mapeamentos cerebrais e classificações diagnósticas ainda são as respostas mais frequentes a este turbilhão de orientações, identidades e preferências sexuais. Este artigo se propõe a debater o tema diversidade sexual, provocando uma reflexão sobre a força do sociocultural que se contrapõe às evidências da biotecnologia.

Palavras-chave: Sexualidade. Gênero. Diversidade Sexual. Diferenças Cerebrais. Transgêneros.

\section{ABSTRACT}

The changes in the field of sexuality during the 2oth century are characterized by the influence of technology, progress in the area of Sociology, Teaching and the creation 
of psychoanalysis. Researches on sexual behavior represented a rupture with clinical sexology studies. The words sex and gender have been and still are used as synonyms, even though belonging to a certain sex means being a man or a woman biologically, whereas gender defines male and female from a behavioral and psychological point of view. On the other hand, gender behavior assumes different meanings in accordance with the culture in which it is inserted. The expression of sexuality, more than being expressed by means of a different biological heritage also constitutes a form of confirmation of social rules, which gives it an ideological character. Brain image techniques extended the knowledge about the male and female brains. Studies show that differences associated with sex happen at different levels of the nervous system, from genetics to behavior, resulting in different male and female brain organization dissociated from stereotypes. The legacy of such changes is unprecedented for the Western civilization. Perplexity, stigmatization, brain mapping and diagnosis classifications are still the most frequent answers to this whirlwind of sexual orientations, identities and preferences. This article proposes to debate the topic of sexual diversity, provoking a reflection on the sociocultural components against the evidences of biotechnology.

Keywords: Sexuality. Gender. Sexual Diversity. Brain Differences. Transgender.

\section{INTRODUÇÃO}

As mudanças no campo da sexualidade ocorridas no século XX foram caracterizadas por grande influência da medicina: especialmente o desenvolvimento das tecnologias reprodutivas (conceptivas e contraceptivas). Por outro lado, progressos na área da Sociologia, Pedagogia, além do advento da psicanálise, avaliaram, ao mesmo tempo em que impactaram o comportamento sexual nesse século, quando o desejo sexual passou a ser valorizado, especialmente para as mulheres.

As pesquisas sobre o comportamento sexual feminino e masculino da população americana, realizadas por Kinsey [23; 24], representaram uma ruptura com os estudos da sexologia clínica do final do século XIX e do início do século XX, focalizados sobre os "desvios sexuais" [18; 25].

Com o advento da Aids, no início dos anos 1980, esses estudos se multiplicaram, contribuindo para explicitar aspectos da sexualidade contemporânea [1; 41].

Nesse campo, a influência da epidemiologia e notadamente da biologia experimental sobre a clínica médica, teve enorme relevância no século XX. Por sua magnitude, afetou também a sexualidade no século XXI [27].

O transplante de órgãos, a pílula anticoncepcional, a barriga de aluguel, a fertilização in vitro, o código genético, o genoma, a clonagem, as células tronco, entre outros, atingiram profundamente as estruturas simbólicas que regem as identificações dos indivíduos (nomeação, filiação, maternidade e paternidade, identidade sexual). Loyola [27] alertou, no início dos anos 2000, que "a liberação do sexo" teria como efeito deixar todo mundo em busca de seu "gênero", de sua identidade genérica e sexual, com cada vez menos respostas possíveis, considerando-se a circulação dos signos e a multiplicidade dos prazeres. Tratava-se, em suma, da transformação das questões de 
identidade sexuada nas de um comércio de signos, de um jogo irônico da indiferença sexual. Esses desafios a sexualidade teria de enfrentar no século XXI" [27].

A partir dessas considerações iniciais, o artigo em pauta passa a debater o tema diversidade sexual, provocando uma reflexão sobre a força das influências socioculturais que se contrapõem às evidências da biotecnologia.

\section{SEXO E GÊNERO}

Não é incomum que as palavras sexo e gênero sejam utilizadas como sinônimos, embora pertencer a determinado sexo signifique ser homem ou mulher, biologicamente, enquanto gênero define o masculino e o feminino, do ponto de vista comportamental e psicológico.

Essa diferença vem sendo muito discutida, na atualidade, desde que a diversidade sexual passou a ser intensamente divulgada, em função das novas formas de comunicação e interação social disponíveis.

O tema não é novo, contudo ainda instiga os pesquisadores e a população geral, ao mesmo tempo em que os estereótipos de masculinidade (força, iniciativa, coragem, competitividade) e os de feminilidade (fragilidade, ternura, necessidade de proteção, interesses domésticos) são questionados, apesar de ainda transmitidos.

Em seu livro "Making sex-body and gender from the Greeks to Freud”, Laqueur [26] enfatiza que, até o século XVIII, o gênero constituía o sexo. As diferenças entre os sexos foram criação do século XX. Até então, o sexo masculino era o único, sendo a mulher um macho incompleto. Diferenças reforçadas instituíram o registro de dois sexos [26].

Também na segunda metade do século passado, os pesquisadores Money e Tucker [32] defendiam que as características biológicas sexuais humanas podem ser assim resumidas: só o homem fecunda; só a mulher menstrua, gesta e amamenta. As outras diferenças entre os sexos não seriam imprescindíveis, mas as quatro funções reprodutoras básicas (fecundação, menstruação, gestação e amamentação) definiriam o sexo de cada um, em qualquer sociedade ou cultura. Segundo essa tese, em todas as sociedades o sexo biológico seria a base de um código regente das interações sociais e da natureza humana. As características sexuais inatas ensejariam elaborações culturais, formando papéis sociais (papéis de gênero), os quais não estariam relacionados com o elemento biológico originário, mas seriam obrigatoriamente decorrência natural dos mesmos. A título de ilustração, vale comentar: a mãe, porque amamenta, também responde pelas tarefas da casa. Deduz-se disso que muitas vezes se creditam às diferenças biológicas o que, na verdade, são acordos entre os sexos, com finalidade funcional e de preservação da espécie [32].

$\mathrm{Na}$ atualidade, o comportamento de gênero assume diferentes significados, conforme a cultura em que se insere. Tais significados podem variar dentro de uma mesma cultura, segundo as conveniências, o que também lhes confere caráter adaptativo/econômico. Em outras palavras, o comportamento do homem e da mulher se adapta para atender, entre outras, às demandas do estilo de vida adotado. Portanto, 
a expressão da sexualidade, muito mais do que se manifestar por diferentes heranças biológicas, também se constitui num meio de confirmação de regras sociais, o que lhe credita caráter ideológico.

\section{DIFERENÇAS CEREBRAIS ENTRE OS SEXOS}

O advento de técnicas de imagem cerebral cada vez mais sofisticadas (ressonância nuclear magnética funcional e tomografia por emissão de pósitrons, por exemplo) ampliou, especialmente na última década, o conhecimento das diferenças do cérebro masculino e feminino sobre as funções cerebrais. Essas descobertas, apesar de reveladoras, são desafiadas por expressões da sexualidade, que nem sempre apresentam correspondência com as respectivas estruturas cerebrais (masculina e feminina).

Em várias regiões do córtex cerebral, a substância cinzenta e a branca diferem, conforme os sexos, não sendo essas diferenças sempre evidentes anatomicamente, mas em caráter funcional, quanto à função dos neurotransmissores ou à resposta metabólica à determinada experiência [3].

Essas particularidades estão associadas à presença ou à ausência de receptores de esteroides sexuais em regiões cerebrais homólogas, durante determinados períodos do desenvolvimento, o que indica a influência de hormônios sexuais sobre as características específicas dos cérebros masculino e feminino [21].

O hipocampo, região cerebral sexualmente dimórfica em sua estrutura e função, está associado ao aprendizado e à memória, às funções neuroquímicas (sistemas serotoninérgico, adrenérgico, colinérgico, entre outros) e à reatividade ao estresse [30]. Há diferenças importantes, sendo essa região maior em mulheres do que em homens. O estresse crônico comprovadamente prejudica as células do hipocampo dos indivíduos do sexo masculino, enquanto esse efeito em mulheres é menos conhecido. Tal vulnerabilidade torna-se fator de risco para o transtorno do estresse pós-traumático e a depressão [31].

A amígdala é maior em homens do que em mulheres, sendo bem conhecido o papel que desempenha na memória para eventos emocionais. O conteúdo emocional da memória (imagens visuais) associa-se à amígdala esquerda em mulheres e à amígdala direita nos homens [9; 10]. Além disso, nas respostas a expressões faciais, a amígdala esquerda responde mais ativamente em mulheres diante das faces que manifestam alegria, padrão oposto ocorre para a amígdala direita [22].

A tomada de decisão depende do córtex pré-frontal. $\mathrm{O}$ desempenho nesta tarefa está mais associado ao hemisfério direito em homens, enquanto associa-se ao hemisfério esquerdo em mulheres [37].

Tomografia por emissão de pósitrons, utilizada para avaliar o nível de síntese de serotonina, encontrou concentração 50\% maior em homens do que em mulheres. Esse resultado ajuda a compreender porque a depressão (que envolve disfunção de serotonina) afeta mais às mulheres do que aos homens, numa proporção de 2 para 1 [33].

Há, ainda, diferenças na responsividade da dopamina ao estímulo dos hormônios sexuais e da anfetamina: mulheres são mais sensíveis para os efeitos reforçadores dos 
psicoestimulantes, o que contribui para que evoluam mais rapidamente à dependência química [29].

Avaliações de competência espacial demonstram que o amadurecimento mais tardio favorece melhor desempenho do que o amadurecimento precoce. Como os meninos tendem a amadurecer mais tarde do que as meninas, essa maturação mais lenta garante vantagem masculina em atividades que exijam habilidade espacial [39].

Portanto, influências associadas ao sexo se dão em todos os níveis sobre o sistema nervoso, desde o genético até o comportamental, resultando em dois mosaicos de organização cerebral - um masculino e outro feminino - aparentemente semelhantes em muitos aspectos [42]. Essas influências são muito importantes, não devendo ser negligenciadas. Mas não favorecem o entendimento da diversidade sexual.

\section{VARIAÇÃO DE GÊNERO NA INFÂNCIA E ORIENTAÇÃO SEXUAL NA VIDA ADULTA}

Estudos têm focado a associação entre variação de gênero (ou comportamento transgênero) na infância e orientação sexual e disforia de gênero na vida adulta. O quanto isto se observa tanto em amostras quanto na população em geral ainda não está definido.

Homossexuais tendem a referir mais história de desconforto em relação ao gênero na infância, quando comparados aos pares heterossexuais $[6 ; 34]$. Por outro lado, a presença na infância de variação de gênero foi associada à orientação homo ou bissexual na vida adulta $[17 ; 40]$. Há indícios de que o comportamento de variação de gênero seja influenciado por exposição cerebral a hormônios sexuais e de que este comportamento seja hereditário $[12 ; 38]$.

Em relação à orientação sexual na vida adulta e à disforia de gênero, diferenças em certas estruturas cerebrais entre os homossexuais e heterossexuais $[2 ; 36]$ e em certas estruturas neuroanatômicas em transgêneros homens-para-mulheres e mulheres-para-homens [20; 28] foram demonstradas.

Quanto à metodologia das pesquisas, as retrospectivas são passíveis de crítica, por viés de recordação. Há, no entanto, um estudo prospectivo que avaliou fatores da infância que podem influenciar a disforia de gênero na adolescência e no adulto jovem. Demonstrou que quanto mais intenso o relato de variação de gênero, maior a chance de persistência dessa variação de disforia de gênero na adolescência [38].

Estudos prospectivos com crianças que sejam representativas da população em geral são necessários. O resultado de um deles, de variação de gênero na infância e orientação sexual e disforia de gênero na vida adulta entre garotos e garotas da população em geral foi recentemente publicado [35]. A variação de gênero na infância e a homossexualidade na vida adulta resultaram associadas na população em geral, mas essa correlação foi mais fraca do que nas amostras clínicas. Vale lembrar que essa associação não é aplicável para a vasta maioria das crianças com variação de gênero e para indivíduos homossexuais e ela também não implica uma relação causal entre esses dois fenômenos [35].

À luz desse achado, pais e cuidadores devem ser informados de que o desfecho 
psicossexual (em termos de orientação sexual e disforia de gênero) não pode ser previsto, pelo conhecimento atual nos dias de hoje. Devem-se evitar ações precipitadas (mudar nomes ou usar pronomes do outro gênero) e se recomendam atitudes mais neutras. Steensma et al. [35] sugerem que os pais devam permitir, por exemplo, que o filho use um vestido em um ambiente protegido, mas não em público, a menos que este desejo transgênero persista, com o passar dos anos. Ter informação sobre o desenvolvimento psicossexual em geral e o esclarecimento de como lidar com esta variação de gênero pode diminuir a pressão dos pais sobre a criança, no sentido de se tornar mais próxima do gênero biológico, sendo mais tolerantes quanto ao comportamento variável do gênero da criança [15].

\section{FUTURO DA DIVERSIDADE SEXUAL}

Tudo indica que a $11^{\mathrm{a}}$ edição da Classificação Internacional de Doenças e Condições Relacionadas à Saúde, da Organização Mundial de Saúde vai "desclassificar" o transexualismo como um transtorno mental e mudar a terminologia de diagnóstico para Incongruência de Gênero. Muito provavelmente irá alterar a colocação da incongruência de gênero, no âmbito da saúde sexual e reprodutiva, como uma categoria separada [16]. Vale lembrar que o Manual Diagnóstico e Estatístico de Transtornos Mentais, $5^{\text {a }}$ edição (DSM-5) "despatologizou” as parafilias (exceto pedofilia, voyeurismo, exibicionismo e frotteurismo), desde que fossem praticadas com consenso entre os parceiros e não causassem sofrimento [5].

Apesar do número de indivíduos com disforia de gênero que frequentam clínicas para avaliação e pessoas que se autodiagnosticam com disforia de gênero/incongruência de gênero estar em franco crescimento ao longo dos últimos anos em muitos países, faltam dados fidedignos sobre a prevalência dessa condição na população geral, de modo a permitir o planejamento dos serviços de saúde [4].

Outra sitação que preocupa é a prevenção de problemas de saúde mental, predominantemente ansiedade, depressão e comportamento de automutilação [11]. Além do discurso de que indivíduos transgêneros são um grupo psicologicamente vulnerável, devem ser implementados programas de acompanhamento que trabalhem o estresse de minorias e a discriminação, o reforço à proteção legal e o apoio social [14].

Estudos prospectivos são escassos e ainda não se tem resposta a respeito de quais os tratamentos com hormônios são mais eficazes e qual é o melhor perfil de efeitos colaterais. Também os níveis de hormônios e por quanto tempo; o papel e o risco de análogos do hormônio liberador de gonadotrofinas $(\mathrm{GnRH})$ e se são superiores aos medicamentos mais antigos (acetato de ciproterona); por quanto tempo os jovens podem usar análogos de GnRH, antes de se introduzir hormônios do sexo oposto e quais são os prós e os contras desta estratégia [4].

$\mathrm{O}$ apoio psicológico, a supressão da puberdade e o funcionamento psicossocial em adolescentes com disforia de gênero são temas relevantes a serem melhor pesquisados. Da mesma forma, sobre cirurgia, há escassez de estudos, quanto aos resultados e comparações de técnicas cirúrgicas reconstrutivas de mamas, dos genitais e 
das cordas vocais [13].

Trabalhar os clínicos para tratar os indivíduos trangêneros como qualquer outra população, não dificultando ou questionando o acesso aos cuidados é outra vertente [8]. A implementação e o desenvolvimento de serviços multidisciplinares para identidade de gênero são fundamentais, de modo a atender todas as idades.

A ênfase em biologia molecular, genética e imagens funcionais cada vez mais atrai investimentos. Tem interesse acadêmico, mas não necessariamente beneficia transgêneros [4]. Estudos bem desenhados, embasados na clínica, podem servir de modelo para futuras e mais amplas pesquisas.

Esclarecimento e educação da população geral (a começar nas escolas) e dos profissionais de Saúde, em particular, são essenciais para a compreensão e a tolerância e para melhor qualidade de vida de indivíduos transgêneros e seus familiares.

\section{CONCLUSÃO}

Uma profusão de recursos tecnológicos, cuja maior acessibilidade se deu a partir do século XX, permitiu que novas manifestações sexuais concretizassem "fantasias" e as divulgassem de forma surpreendentemente eficiente, por meio de variados canais midiáticos, especialmente a partir do final do século passado.

Na sequência, a diversidade sexual veio a público, numa dimensão inédita.

O legado dessa mudança sem precedentes para a civilização ocidental ainda não foi calculado.

Ainda a perplexidade, a estigmatização, os mapeamentos cerebrais e as classificações diagnósticas são as respostas mais frequentes a este turbilhão de orientações, identidades e preferências sexuais.

\section{REFERÊNCIAS}

[1] ALBARRACÍN, D., et al. Persuasive communications to change actions: an analysis of behavioral and cognitive impact in HIV prevention. Health Psychology, v. 22, n. 2, p. 166-177, 2003. DOI: 10.1037/0278-6133.22.2.166.

[2] ALLEN, L. S., GORSKI, R. A. Sexual orientation and the size of the anterior commissure in the human brain. Proceedings of the National Academy of Sciences of the United States of America, v. 89, n. 15, p. 7199-7202, 1992. PNAS 1992 89: 7199-7202.

[3] ALLEN, J. S., et al. Sexual dimorphism and asymmetries in the gray-white composition of the human cerebrum. Neuroimage, v. 18, n. 880-894, 2003. DOI:10.1016/S1053-8119(03)00034-X.

[4] ARCELUS, J., BOUMAN, W. P. Current and future direction of gender dysphoria and gender incongruence research. The Journal of Sexual Medicine, v. 12, n. 12, p. 2226-2228, 2015. DOI: 10.1111/jsm.13065.

[5] ASSOCIAÇÃO PSIQUIÁTRICAAMERICANA(APA). Manual diagnóstico 
e estatístico de transtornos mentais. $5^{\mathrm{a}}$ ed. Porto Alegre: Artmed, 2013.

[6] BAILEY, J. M., ZUCKER, K. J. Childhood sex-typed behavior and sexual orientation: A conceptual analysis and quantitative review. Developmental Psychology, v. 31, n. 1, p. 43-55, 1995.

[7] BIBER, C. L., et al. A study of sex differences in presentation for care of HIV. AIDS Patient Care STDS, v. 13, n. 2, p. 103-110, 1999. DOI: 10.1089/ apc.1999.13.103.

[8] BOUMAN, W.P., et al. Yes and yes again: Are standards of care which require two referrals for genital reconstructive surgery ethical? Sexual and Relationship Therapy, v. 29, n. 4, p. 377-389, 2014. DOI: 10.1080/14681994.2014.954993.

[9] CAHILL, L., et al. Sex-related difference in amygdala activity during emotionally influenced memory storage. Neurobiology of Learning and Memory, v. 75, n. 1, p. 1-9, 2001. DOI: 10.1006/nlme.2000.3999.

[10] CAHILL, L., et al. Sex-related hemispheric lateralization of amygdala function in emotionally influenced memory: an FMRI investigation. Learning \& Memory, v. 11, n. 3, p. 261-266, 2004. DOI: 10.1101/lm.70504.

[11] CLAES, L., et al. Non-suicidal self-injury in trans people: Associations with psychological symptoms, victimization, interpersonal functioning and perceived social support. The Journal of Sexual Medicine, v. 12, n. 1, p. 168-179, 2015. DOI: $10.1111 /$ jsm.12711.

[12] COHEN-BENDAHAN, C. C., VAN DE BEEK, C., BERENBAUM, S. A. Prenatal sex-hormone effect on child and adult sex-typed behavior: Methods and findings. Neuroscience and Biobehavioral Reviews, v. 29, n. 2, p. 353-384, 2005. DOI: 10.1016/j.neubiorev.2004.11.004.

[13] COSTA, R., et al. Psychological support, puberty suppression, and psychosocial functioning in adolescents with gender dysphoria. The Journal of Sexual Medicine, v. 12, n. 11, p. 2206-2214, 2015. DOI: http://dx.doi.org/10.1111/ jsm.13034.

[14] DAVEY, A., et al. Social support and psychological wellbeing: A comparison of patients with gender dysphoria and matched controls. The Journal of Sexual Medicine, v. 11, n. 12, p. 2976-2985, 2014. DOI: 10.1111/jsm.12681.

[15] DE VRIES, A. L., COHEN-KETTENIS, P. T. Clinical management of gender dysphoria in children and adolescents: The Dutch approach. Journal of Homosexuality, v. 59, n. 3, p. 301-320, 2012. DOI: 10.1080/00918369.2012.653300.

[16] DRESCHER, J., COHEN-KETTENIS, P., WINTER, S. Minding the body: Situating gender identity diagnoses in the ICD-11. International Review of Psychiatry, v. 24, n. 6, p. 568-577, 2012. DOI: 10.3109/09540261.2012.741575.

[17] DRUMMOND, K. D., et al. A follow-up study of girls with gender identity disorder. Developmental Psychology, v. 44, n. 1, p. 34-45, 2008. DOI: 10.1037/0012-1649.44.1.34.

[18] ELLIS, H. Studies in the psychology of sex. New York: Randon House, 1936.

[19] FLORENCE, E., et al., EUROSUPPORT STUDY GROUP. Prevalence and factors associated with sexual dysfunction among HIV-positive women in Europe. AIDS Care, v. 16, n. 5, p. 550-557, 2004. DOI: 10.1080/09540120410001716333. 
[20] GARCIA-FALGUERAS, A., SWAAB, D. F. A sex difference in the hypothalamic uncinate nucleus: Relationship to gender identity. Brain, v. 131, n. Pt 12, p. 3132-3146. 2008. DOI: 10.1093/brain/awn276.

[21] GOLDSTEIN, J. M., et al. Normal sexual dimorphism of the adult human brain assessed by in vivo magnetic resonance imaging. Cerebral Cortex, v. 11, n. 6, p. 490-497, 2001. DOI: 10.1093/cercor/11.6.490.

[22] KILLGORE, W., YURGELUN-TODD, D. Sex differences in amygdala activation during the perception of facial affect. Neuroreport, v. 12, n. 11, p. 2543-2547, 2001. DOI: 10.1097/00001756-200108080-00050.

[23] KINSEY, A. S., et al. Sexual behavior in the human male. Philadelphia: Saunders, 1948.

[24] KINSEY, A. S., MARTIN, C. R., GEBBARD, P. H. Sexual behavior in the human female. Philadelphia: Saunders, 1954.

[25] KRAFFT-EBING, R. Psychopathia sexualis. Paris: Payot, 1950.

[26] LAQUEUR, T. La construcción del sexo: cuerpo x género desde los griegos hasta Freud. Madrid: Cátedra, 1994.

[27] LOYOLA, M. A. Sexualidade e medicina: a revolução do século XX. Cadernos de Saúde Pública, v. 19, n. 4, p. 875-884, 2003. DOI: 10.1590/ So102-11X2003000400002.

[28] LUDERS, E., et al. Regional gray matter variation in male-to-female transsexualism. Neuroimage, v. 46, n. 4, p. 904-907, 2009. DOI: 10.1016/j. neuroimage.2009.03.048.

[29] LYNCH, W. J., ROTH, M. E., CARROLL, M. E. Biological basis of sex differences in drug abuse: preclinical and clinical studies. Psychopharmacology (Berlin), v. 164, n. 2, p. 121-137, 2002. DOI: 10.1007/soo213-002-1183-2.

[30] MADEIRA, M. D., LIEBERMAN, A. R. Sexual dimorphism in the mammalian limbic system. Progress in Neurobiology, v. 45, n. 4, p. 275-333, p. 1995. DOI: 10.1016/0301-0082(94)00052-J.

[31] MCEWEN, B. S. The neurobiology of stress: from serendipity to clinical relevance. Brain Research, v. 886, n. 1-2, p. 172-189, 2000. DOI: 10.1016/ Sooo6-8993(00)02950-4.

[32] MONEY, J., TUCKER, P. Os papéis sexuais. São Paulo: Brasiliense, 1981.

[33] NISHIZAWA, S., et al. Differences between males and females in rates of serotonin synthesis in human brain. Proceedings of the National Academy of Sciences of the United States of America, v. 94, n. 10, p. 5308-5313, 1997.

[34] SMITH, Y. L., et al. Transsexual subtypes: Clinical and theoretical significance. Psychiatry Research, v. 137, n. 3, p. 151-160, 2005. DOI: 10.1016/j. psychres.2005.01.008.

[35] STEENSMA, T. D., et al. Gender variance in childhood and sexual orientation in adulthood: A prospective study. The Journal of Sexual Medicine, v. 10, n. 11, p. 2723-2733, 2013. DOI: 10.1111/j.1743-6109.2012.02701.x.

[36] SWAAB, D. F., et al. Sexual differentiation of the human hypothalamus: Differences according to sex, sexual orientation, and transsexuality. In: ELBERTZ, L. (Ed.). Sexual orientation: Toward biological understanding. Westport, 
CT: Publishers/Greenwood Publishing Group, Inc.; p. 129-150, 1997. DOI: 10.1080/09513590400018231.

[37] TRANEL, D., et al. Does gender play a role in functional asymmetry of ventromedial prefrontal cortex? Brain, v. 128, n. Pt 12, p. 2872-2881, 2005. DOI: 10.1093/brain/awh643.

[38] VAN BEIJSTERVELDT, C. E., HUDZIAK, J. J., BOOMSMA, D. I. Genetic and environmental influences on cross-gender behavior and relation to behavior problems: A study of Dutch twins at ages 7 and 10 years. Archives of Sexual Behavior, v. 35, n. 6, p. 647-658, 2006. DOI: 10.1007/s10508-006-9072-0.

[39] WABER, D. P. Sex differences in mental abilities, hemispheric lateralization, and rate of physical growth in adolescence. Developmental Psychology, v. 13, n. 1, p. 29-38, 1977. DOI: 10.1037/oo12-1649.13.1.29.

[40] WALLIEN, M. S., COHEN-KETTENIS, P. T. Psychosexual outcome of gender-dysphoric children. Journal of the American Academy of Child and Adolescent Psychiatry, v. 47, n. 12, p. 1413-1423. 2008. DOI: 10.1097/ CHI.obo13e31818956b9.

[41] WINGOOD, G. M., DICLEMENTE, R. J. HIV sexual risk reduction interventions for women: a review. American Journal of Preventive Medicine, $v$. 12, n. 3, p. 209-217, 1996.

[42] WITELSON, S. F. Neural sexual mosaicism: sexual differentiation of the human temporo-parietal region for functional asymmetry. Psychoneuroendocrinology, v. 16, n. 1-3, p. 131-153, 1991. DOI: 10.1016/0306-4530(91)90075-5.

[43] ZUCKER, K. J., et al. The recalled childhood gender identity/gender role questionnaire: Psychometric properties. Sex Roles, v. 54, n. 7, p. 469-83, 2006. DOI: 10.1007/s11199-006-9019-x.

CARMITA H. N. ABDO psiquiatra, livre-docente e professora associada do Departamento de Psiquiatria da Faculdade de Medicina da Universidade de São Paulo (FM-USP), coordenadora do Programa de Estudos em Sexualidade (ProSex) do Instituto de Psiquiatria do Hospital das Clínicas da FM-USP - e-mail: carmita.abdo@uol.com.br. 